\title{
Kosten des Klimawandels und Auswirkungen auf die Wirtschaft
}

Gernot Klepper, Wilfried Rickels, Oliver Schenker, Reimund Schwarze,

Hubertus Bardt, Hendrik Biebeler, Mahammad Mahammadzadeh, Sven Schulze

25.1 Herausforderungen für die Quantifizierung

der Kosten des Klimawandels - 254

25.1.1 Wirtschaftliche Kosten und soziale Kosten - 254

25.1.2 Kosten auf unterschiedlichen Zeitskalen - 254

25.1.3 Systemische Wirkungen des Klimawandels - 255

25.1.4 Unsicherheiten in Bezug auf Extremereignisse - 255

25.1.5 Projektionen von Anpassungsreaktionen - 255

25.2 Kosten des Klimawandels: Modellierungsansätze - 255

25.3 Wirtschaftliche Auswirkungen des Klimawandels in Deutschland - 256

25.4 Abschätzung sektoraler Kosten des Klimawandels - 259

25.4.1 Hochwasser- und Küstenschutz - 259

25.4.2 Hitzewellen und Gesundheitskosten - 260

25.5 Exkurs: Subjektive Einschätzung der Betroffenheit von Unternehmen - 262

$25.6 \quad$ Kurz gesagt -263

Literatur - 263 
Die Bestimmung der Kosten und die Bewertung der wirtschaftlichen Auswirkungen des Klimawandels und möglicher Anpassungsmaßnahmen sind komplex. Klimawandelbedingte Kosten entstehen in einer Kaskade von Wirkungsmechanismen und -kreisläufen, die jeweils mit zahlreichen Unsicherheiten verbunden sind. Die Menge der emittierten Treibhausgasemissionen bestimmt, wie sich die Atmosphäre und damit das Klima auf der Erde verändert. Die Reaktion des Klimasystems mit seinen zahlreichen Rückkopplungseffekten bestimmt die regional stark variierenden Klimaveränderungen. Diese Veränderungen gehen einher mit Veränderungen der verschiedenen Erdsysteme wie z. B. der Wachstumsbedingungen für Pflanzen oder der Wasserkreisläufe, die ihrerseits wieder auf die Zusammensetzung der Atmosphäre sowie auf die (regionale) Klimareaktion rückwirken. Die Veränderung des regionalen Klimas inklusive veränderter interner Variabilität von Extremereignissen hat Wirtschafts- und Wohlfahrtseffekte, die sowohl positiv als auch negativ ausfallen können. Die Reaktion auf diese Effekte durch Emissionskontrolle und Anpassung hat wiederum einen direkten Einfluss auf den Wirkungskreislauf, weil durch sie die Menge der Treibhausgasemissionen bestimmt wird.

Globale und regionale Klimaprojektionen für Deutschland wurden in Teil 1 diskutiert, und die vorangegangenen Kapitel in diesem Teil stellen regionale Besonderheiten sowie sektorale Auswirkungen des Klimawandels detailliert dar. In diesem Kapitel werden Möglichkeiten und Grenzen der gesamtwirtschaftlichen Bewertung beschrieben; potenzielle Probleme, Herausforderungen und Implikationen werden exemplarisch für den Bereich Gesundheit sowie Küstenschutz diskutiert. Darüber hinaus bietet das Kapitel einen Überblick über die Einschätzung von Unternehmen zu den Auswirkungen des Klimawandels.

\subsection{Herausforderungen für die Quantifizierung der Kosten des Klimawandels}

Klimawandel in seinen vielen regionalen Facetten verändert das komplexe Geflecht von Produktionsmöglichkeiten und Lebensqualität. Die Anpassung der Menschen an diese Veränderungen wird durch die regionalen Anpassungsoptionen bestimmt. Dies schließt individuelle Anpassung von Konsumenten und Unternehmen ein, aber es betrifft auch staatliche Maßnahmen, die eine Anpassung an die Auswirkungen des Klimawandels unterstützen. Diese Anpassungsprozesse werden auch durch Rückkopplungsprozesse von Klimawandel in anderen Weltregionen beeinflusst. So können internationale Handelsströme und globale Wertschöpfungsketten verändert werden. Eine weitere Anpassungsreaktion, die auf eine Region wie Deutschland einwirken kann, ist die Migration aus Regionen, deren Lebensgrundlagen durch Klimawandel besonders beeinträchtigt werden, in Regionen, in denen der Klimawandel nicht so starke negative Auswirkungen hat oder sogar die wirtschaftlichen Möglichkeiten verbessert. Aus diesen globalen Wechselwirkungen ergeben sich neben den direkten Auswirkungen zusätzliche indirekte Auswirkungen des Klimawandels. Eine Abschätzung der Kosten des Klimawandels für Deutschland erfordert daher die Abschätzung dieser direkten und indirekten Effekte. Die Abschätzung der (wirtschaftlichen) Rückkopplungseffekte und damit der indirekten Kosten ist mit großen Unsicherheiten und Ungewissheiten verbunden, weshalb die Betrachtungen in den vorangegangenen Kapiteln in Teil III insbesondere auf die direkten Auswirkungen des Klimawandels in Deutschland fokussieren.

Eine Abschätzung von Kosten des Klimawandels ist mit einer Reihe weiterer Herausforderungen konfrontiert, die eine Quantifizierung erschweren und unter den folgenden Stichworten zusammengefasst werden können:

- Wirtschaftliche Kosten und soziale Kosten.

- Kosten auf unterschiedlichen Zeitskalen.

- Systemische Wirkungen des Klimawandels.

- Unsicherheiten in Bezug auf Extremereignisse.

- Projektionen von Anpassungsreaktionen.

\subsubsection{Wirtschaftliche Kosten und soziale Kosten}

Der Klimawandel wird Wirtschaftsprozesse direkt beeinflussen. Diese Veränderungen können im Prinzip mithilfe von Modellsimulationen wirtschaftlicher Prozesse quantifiziert werden. Gleichzeitig treten Veränderungen auf, die nicht direkt die Wirtschaftsaktivitäten beeinflussen, sondern das Wohlbefinden der Menschen. Diese (zusätzlichen) Wohlfahrtseinbußen lassen sich nur schwer quantifizieren und müssen indirekt bewertet werden, indem beispielsweise die subjektiv empfundenen Kosten erfragt werden. Dies betrifft z. B. die unten beschriebenen Effekte von Hitzewellen (Definition $>$ Kap. 6), bei denen nur die direkten wirtschaftlichen Auswirkungen, nicht aber die Verluste an Lebensqualität erfasst wurden. Noch extremer wird die Herausforderung, wenn der Klimawandel zu Todesfällen führt, die als Teil der Kosten identifiziert werden sollen. Daraus ergeben sich nicht zuletzt ethische Kontroversen.

\subsubsection{Kosten auf unterschiedlichen Zeitskalen}

Die Kosten des Klimawandels werden in dem Maße steigen, in dem sich das Klima zunehmend stärker verändert. Konkret bedeutet dies, dass bis zur Mitte dieses Jahrhunderts die Auswirkungen weitaus geringer ausfallen werden als gegen Ende des Jahrhunderts ( Kap.4). Das hat zur Konsequenz, dass die Kosten des Klimawandels in der zweiten Hälfte dieses Jahrhunderts vor dem Hintergrund der dann vorherrschenden wirtschaftlichen Situation, sowohl in Deutschland als auch weltweit, bestimmt werden müssten. Allerdings gibt es praktisch keine Vorstellung darüber, wie die deutsche Wirtschaft und die Weltwirtschaft sich in den nächsten 50 Jahren entwickeln werden. Ein wichtiger Faktor für die Bestimmung der Kosten des Klimawandels ist natürlich das Ausmaß der Emissionen bzw. des Klimaschutzes, der ja den Klimawandel bestimmt. 


\subsubsection{Systemische Wirkungen des Klimawandels}

Der Klimawandel hat vielfältige Ausprägungen und betrifft alle Lebensbereiche, direkt oder zumindest indirekt alle Wirtschaftsaktivitäten und verursacht gesellschaftliche Anpassungsprozesse. Die Summe dieser Effekte und ihrer miteinander reagierenden Rückkopplungseffekte kann heute nicht in angemessener Weise in Simulationsmodellen für die nächsten Jahrzehnte oder gar bis zum Ende dieses Jahrhunderts abgebildet werden. Der Klimawandel selbst wird darüber hinaus gesellschaftliche Reaktionen in Bezug auf Vermeidungsmaßnahmen von Treibhausgasemissionen hervorrufen, die wiederum die Kosten des Klimawandels verändern.

Erste Schritte in der Quantifizierung der wirtschaftlichen Folgen des Klimawandels bestehen deshalb darin, sich in der Forschung auf bestimmte Phänomene des Klimawandels zu konzentrieren, etwa auf Hitzewellen und bestimmte Wirtschaftssektoren, oder Regionen auf ihre Anfälligkeit demgegenüber zu untersuchen, etwa Landwirtschaft ( Kap. 18), Küstenzonen oder Tourismus ( Kap. 23). Die verschiedenen sektoral geschätzten Kosten können aber nicht unbedingt addiert werden, um zu den gesamtwirtschaftlichen Kosten zu kommen, denn dadurch würden positive wie auch negative Rückkopplungseffekte ignoriert.

\subsubsection{Unsicherheiten in Bezug auf Extremereignisse}

Für Deutschland wird vermutlich die Zunahme der Häufigkeit von Extremereignissen eine wichtige Rolle spielen. Die Extremereignisse sind von der Seite der naturwissenschaftlichen Modellierung her schon schwer zu quantifizieren. Noch schwieriger ist dies bei den wirtschaftlichen Folgen. Das Wissen um die Zunahme wird höchstwahrscheinlich zu Vorsorgemaßnahmen führen, die die Kosten der Extremereignisse verringern sollen. Darüber hinaus wird der Umfang dieser Vorsorgemaßnahmen entscheidend durch gesellschaftliche und rechtliche Prozesse determiniert. Nicht zuletzt beinhalten die Entscheidungen über die Vorsorge gegenüber Extremereignissen auch eine moralische Bewertung der Akzeptanz von Risiken.

\subsubsection{Projektionen von Anpassungsreaktionen}

Während der Klimawandel aufgrund der langen Verweildauer vieler Treibhausgase in der Atmosphäre frühzeitiges Handeln zur Emissionsvermeidung verlangt, gibt es Anpassungsmaßnahmen, die - anders als bei den Klimaschutzanstrengungen - zeitnah die Schäden größerer Klimawandelfolgen reduzieren können. Das hat zur Folge, dass Projektionen derartiger Maßnahmen sich auf die zweite Hälfte dieses Jahrhunderts konzentrieren, wenn die Anpassungsmaßnahmen besonders wichtig werden. Die wirtschaftlichen und gesellschaftlichen Rahmenbedingungen für Anpassung für diesen entfernten Zeitraum sind allerdings heute kaum verlässlich in Projektionen abbildbar. Daneben gibt es eine Reihe von Vorsorgeinvestitionen, die schon frühzeitig in Angriff genommen werden sollten. Dies trifft für Infrastrukturen zu, die eine lange Lebensdauer besitzen.

\subsection{Kosten des Klimawandels: Modellierungsansätze}

Die wirtschaftliche Bewertung des Klimawandels erfordert eine integrierte Betrachtung von natürlichen Veränderungen des Erdsystems und damit einhergehenden wirtschaftlichen Wirkungszusammenhängen. Angesichts der komplexen Wirkungszusammenhänge konzentrieren sich diese integrierten Bewertungsmodelle (Integrated Assessment Models, IAMs) auf bestimmte Aspekte von Wirkungskaskaden und Rückkopplungseffekten. Vereinfacht kann man zwischen IAMs mit exogenen und endogenen Emissionspfaden unterscheiden:

- Bei IAMs mit exogenen Emissionspfaden werden unterschiedliche Emissions- und damit Klimawandelszenarien detailliert bewertet (Szenarienanalyse).

- Bei IAMs mit endogenen Emissionspfaden werden die „optimalen“ Emissionen als Reaktion auf den Klimawandel durch die Emissionskontrolle bestimmt (Optimierungsmodelle).

Szenarienanalysen basieren auf vorgegebenen naturwissenschaftlichen Klimaszenarien und bewerten daher exogene Emissionspfade. Die derzeit untersuchten Emissionspfade sind aus den RCP-Szenarien abgeleitet, die im Zuge des Fünften Sachstandsberichts des Weltklimarats (IPCC) die vorangegangene Generation von SRES-Emissionsszenarien abgelöst haben (van Vuuren et al. 2011; O’Neill et al. 2013). Bei den Szenarienanalysen werden unterschiedliche regionale und sektorale Fokussierungen vorgenommen sowie deren Interaktionen berücksichtigt. Rein sektorale Studien versuchen, die direkten Kosten des Klimawandels für bestimmte Wirtschaftssektoren oder Handlungsfelder zu bestimmen, ignorieren dabei aber gesamtwirtschaftliche Rückkopplungseffekte. Regional fokussierte Analysen integrieren häufig gesamtwirtschaftliche Rückkopplungseffekte, berücksichtigen aber nicht die indirekten Effekte des Klimawandels im Rest der Welt.

Rückkopplungseffekte zwischen den Kosten des Klimawandels einschließlich der Anpassungsmaßnahmen und den Kosten des Klimaschutzes sind Grundlage für „optimale“ Emissionspfade, deren Berechnung in Optimierungsmodellen allerdings stark vereinfachte Wirkungsketten und Auswirkungsbeschreibungen voraussetzt. Diese werden ihrerseits in stilisierter Form aus Ergebnissen von wirtschaftlichen Szenarienanalysen abgeleitet. Eine Bewertung der wirtschaftlichen Auswirkungen des Klimawandels sowie die Bestimmung des wirtschaftlich effizienten Klimaschutzes setzen daher die Verwendung und Entwicklung beider Modellgruppen voraus. Allerdings hat die Forschung sich sehr viel stärker auf die globalen IAMs konzentriert als auf die sektoralen und regionalen Analysen, auf denen diese IAMs aufbauen.

Die Optimierungsmodelle benutzen in der Regel hoch aggregierte Schadensfunktionen, die im Extremfall den volkswirtschaftlichen Schaden des Klimawandels als funktionalen 


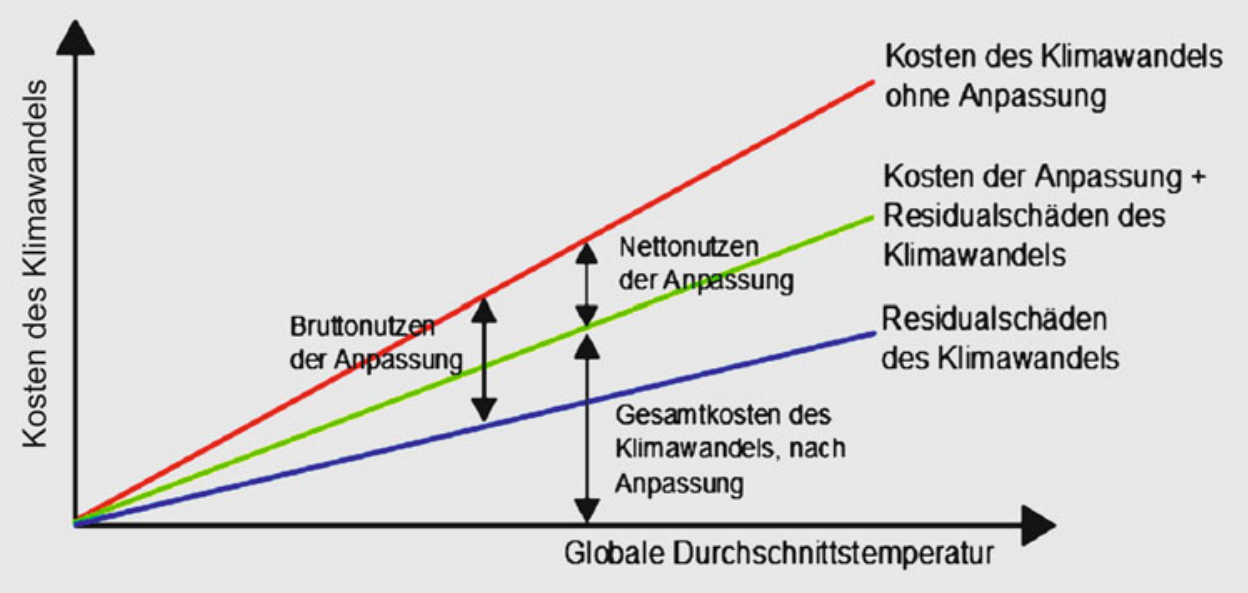

- Abb. 25.1 Regionale Kosten des Klimawandels mit und ohne Anpassung (angelehnt an Stern 2007). Aus Gründen der Vereinfachung ist die Abhängigkeit der Kosten des Klimawandels von der globalen Durchschnittstemperatur linear dargestellt; in der Realität ist aber von einem deutlich nichtlinearen, konvexen Verlauf auszugehen
Zusammenhang von Temperaturänderung und Sozialprodukt definieren, meist in einer nichtlinearen Beziehung (Pindyck 2013; Fisher-Vanden et al. 2013). Das bekannteste Modell dieser Art ist das DICE-Modell von Nordhaus (Nordhaus 1991, 2010, 2014; Nordhaus und Yang 1996), das in vielen Varianten weiterentwickelt worden ist. Durch ihren Fokus auf die lange zeitliche Dimension sind diese Modelle in ihrer ökonomischen Struktur meist relativ einfach gehalten.

Das hohe Aggregationsniveau der Optimierungsmodelle begrenzt die Möglichkeit einer detaillierten Darstellung regionaler Anpassungsmöglichkeiten und hat dadurch möglicherweise einen beträchtlichen Einfluss auf die Abschätzung der Kosten des Klimawandels. In den meisten Studien ist Anpassung als Reaktion auf Klimafolgen nur implizit innerhalb der Schadensfunktion enthalten. Meist wird hierzu angenommen, dass sich die betroffenen Akteure autonom aus Eigeninteresse kosteneffizient an Klimafolgen anpassen würden. Auf dieser Annahme basiert auch die kleine Anzahl an Modellen, die Anpassung als Kontrollvariable explizit modellendogen beinhaltet. AD-DICE, ein Derivat des DICE-Modells, modelliert Anpassung als sogenannte Stromgröße (de Bruin et al. 2009). Das heißt, Kosten und Nutzen von Anpassungsmaßnahmen fallen gleichzeitig an. Bosello et al. (2010) wählen in ihren Arbeiten mit dem AD-WITCH-Modell einen anderen Ansatz und modellieren Anpassung als Bestandsgröße, in die investiert werden muss, damit es sich später auszahlt. Beide Ansätze sind plausibel für bestimmte Anpassungsmaßnahmen, können aber nicht die gesamte Komplexität von Anpassung abbilden.

Globale Optimierungsmodelle haben meistens eine zu grobe räumliche Abbildung, um explizit Ergebnisse für Deutschland ablesen zu können; wohl aber lassen sich Ergebnisse für Nordoder Westeuropa ablesen. Wie aber bereits erwähnt, bieten diese Optimierungsmodelle in Bezug auf die sektoralen Auswirkungen kein sehr detailliertes Bild. Für relativ kleine Wirtschaftsräume wie Deutschland, bei dem die Rückkopplung der Emissionsvermeidung auf den globalen Klimawandel vernachlässigbar ist, bieten sich Szenarienanalysen an, die eine detaillierte Abbildung der wirtschaftlichen Auswirkungen untersuchen können.

Wirtschaftliche Szenarienanalysen ermöglichen außerdem eine genauere Untersuchung, inwieweit Anpassungsmaßnahmen die Auswirkungen den Klimawandels abschwächen können. Für die Betrachtung der Anpassungsmaßnahmen ist es hilfreich, zwischen verschiedenen Kostenkategorien zu unterscheiden:

- Kosten des Klimawandels ohne Anpassungsmaßnahmen,

- Kosten der Maßnahmen zur Anpassung an den Klimawandel und

- Kosten des Klimawandels nach der Umsetzung von Anpassungsmaßnahmen (Residualschäden).

Abb. 25.1 stellt vereinfacht dar, wie anhand dieser Unterscheidung verschiedene Dimensionen der Kosten identifiziert werden können. Natürlich sind die zu betrachtenden Kostengrößen nicht, wie in der Abbildung vereinfacht dargestellt, linear und durch die globale Durchschnittstemperatur bestimmt, sondern durch unterschiedliche regionale und sektorale Klimaparameter wie Hitze- oder Niederschlagsextreme, die die Schäden sprunghaft nach oben treiben können.

\subsection{Wirtschaftliche Auswirkungen des Klimawandels in Deutschland}

Der Weltklimarat nimmt in seinem Fünften Sachstandsbericht (IPCC 2014) eine umfangreiche und detaillierte Klassifizierung der regionalen Risiken und Auswirkungen vor, inklusive der Bewertung, wie und in welchem Ausmaß diese Auswirkungen durch Vermeidung und Anpassung abgeschwächt werden können. Ersteres wird durch die oben angesprochenen RCP-Emissionsszenarien abgebildet; die Anpassungsmöglichkeiten werden durch eine Abschätzung der prozentualen Reduktion der Auswirkungen (für jedes Szenario) durch Anpassung dargestellt. Der IPCC vermeidet allerdings eine monetäre Bewertung seiner Einschätzung und präsentiert insofern nur eine qualitative Einschätzung der Auswirkungen, indem er für jede Region die wesentlichen Risiken darstellt. - Abb. 25.2 zeigt die IPCC Einschätzung der wesentlichen Risiken für Europa.

- Abb. 25.2 zeigt die Einschätzung für zwei Emissionsszenarien, die in der langen Frist (2080-2100) entweder zu einem Anstieg der globalen Durchschnittstemperatur um $2{ }^{\circ} \mathrm{C}$ (relativ starke Emissionsvermeidung) oder $4^{\circ} \mathrm{C}$ (geringe Emissionsver- 
Europa

\begin{tabular}{|c|c|c|c|c|}
\hline \multicolumn{5}{|c|}{ Europa } \\
\hline \multirow{5}{*}{ 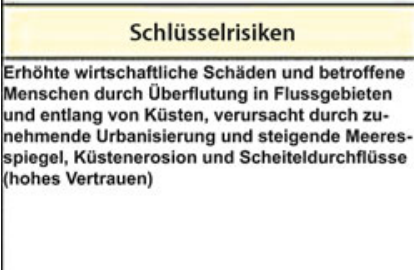 } & \multirow{5}{*}{\begin{tabular}{|l}
\multicolumn{1}{|c}{ Anpassung - Probleme und Perspektiven } \\
Anpassung kann die meisten der projizierten Schäden verhindern \\
(hohes Vertrauen) \\
-Signifikante Erfahrungen mit rein technischen Lösungen zum Hochwasser- \\
schutz und zunehmende Erfahrung mit Revitalisierung von Auen \\
-Hohe Kosten für verstärkten Hochwasserschutz \\
-Potenzielle Hemmnisse für die Umsetzung: Starke Nachfrage nach Land sowie \\
Konflikte mit Umwelt- und Landschaftsschutzzielen
\end{tabular}} & \multirow{5}{*}{$\begin{array}{c}\text { Klimatische } \\
\text { Antriebskräfte }\end{array}$} & Zeitrahmen & $\begin{array}{c}\text { Risiko \& } \\
\text { Anpassungspotenzial }\end{array}$ \\
\hline & & & & Mittel \\
\hline & & & Gegenwart & $10 / 2 /$. \\
\hline & & & \begin{tabular}{|c|} 
Kurzfristig \\
$(2030-2040)$ \\
\end{tabular} & VIII/ \\
\hline & & & $\begin{array}{r}\text { Langfristig } 2^{\circ} \mathrm{C} \\
(2080-2100){ }^{\circ} \mathrm{C} C\end{array}$ & $\begin{array}{l}\text { WIII } \\
\text { TIII }\end{array}$ \\
\hline \multirow{4}{*}{ 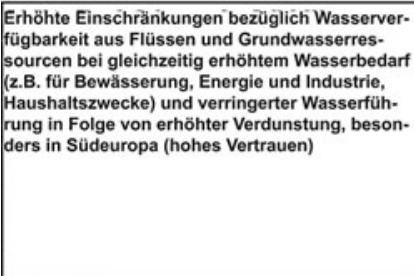 } & \multirow{4}{*}{$\begin{array}{l}\text { - Bekannte Potenziale für die Anpassung durch größere Effizienz in der Wasser- } \\
\text { nutzung und Strategien zur Einsparung von Wasser (z.B. bei Bewässserung, An- } \\
\text { bau von angepassten Nutzpplanzen, Landnutzung, Industrien, Haushaltszwecke) } \\
\text {-Umsetzung von bewährten Methoden und politischen Steuerungsinstrumenten } \\
\text { in Managementplänen für Flusseinzugsgebiete und integriertes Wassermanage- } \\
\text { ment }\end{array}$} & \multirow{4}{*}{ 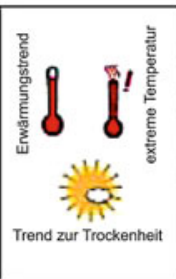 } & & $\begin{array}{c}\text { Sehr } \\
\text { niedrig }\end{array}$ \\
\hline & & & Gegenwart & I/ \\
\hline & & & \begin{tabular}{|c|} 
Kurzfristig \\
$(2030-2040)$ \\
\end{tabular} & $V / 2$ \\
\hline & & & $\begin{array}{r}\text { Langfristig } 2^{\circ} \mathrm{C} \\
(2080-2100) \\
4^{\circ} \mathrm{C}\end{array}$ & $\begin{array}{l}Y / / \\
\square / D / 2\end{array}$ \\
\hline \multirow{5}{*}{$\begin{array}{l}\text { Erhöhte wirtschaftliche Schăden und gesund- } \\
\text { heittiche Beeinträchtigung durch Hitzewellen } \\
\text { verbunden mit Folgen für Gesundheit und } \\
\text { Wohlbefinden, Arbeitsproduktivităt, Ernteertrăge } \\
\text { Luftqualitats und zunehmente Risiken von Wald- } \\
\text { und Flächenbränden in Südeuropa und im borea } \\
\text { len Gebiet von Russland (mittleres Vertrauen) }\end{array}$} & \multirow{5}{*}{$\begin{array}{l}\text {-Einsatz von Frühwarnsystemen } \\
\text {-Anpassung von Wohnstätten und Arbeitsplätzen und der Infrastruktur für Trans- } \\
\text { port und Energie } \\
\text {-Verringerung der Emissionen um die Luftqualität zu verbessern } \\
\text {-Verbesserte Bekămpfung von Wald- und Flächenbränden } \\
\text {-Entwicklung von Versicherungsprodukten gegen durch Unwetter verursachte } \\
\text { Ernteausfälle }\end{array}$} & \multirow{5}{*}{$\int_{\text {extreme Temperatur }}^{\text {no }}$} & & \begin{tabular}{|l} 
Sehr \\
niedrig \\
\end{tabular} \\
\hline & & & Gegenwart & I \\
\hline & & & \begin{tabular}{|c|} 
Kurzfristig \\
$(2030-2040)$ \\
\end{tabular} & $1 / 2$ \\
\hline & & & Langfristig $2^{\circ} \mathrm{C}$ & / \\
\hline & & & & $7 D I$ \\
\hline
\end{tabular}

- Abb. 25.2 Einschätzung der wesentlichen Risiken des Klimawandels und Möglichkeiten zur Anpassung bezogen auf Europa, basierend auf IPCC 2014. Bei den langfristigen Auswirkungen (2080 bis 2100) wird zwischen zwei Emissionsszenarien unterschieden (Temperaturanstieg um $2^{\circ}$ und $4^{\circ} \mathrm{C}$ ). Die schraffierte Fläche zeigt jeweils, inwieweit sich die Auswirkungen durch Anpassung abschwächen lassen. (IPCC 2014, SPM.2 Tab. 1, Ausschnitte)

meidung) führen. Als wesentliche Risiken identifiziert der IPCC vermehrte Überschwemmungen, verschärfte Wasserknappheit (insbesondere in Südeuropa) und eine größere Häufigkeit von Hitzewellen. Vor allem bei der Wasserknappheit und den Auswirkungen von Hitzewellen wird das Potenzial, deren (wirtschaftliche) Auswirkungen durch Anpassungsmaßnahmen abzuschwächen, als eher gering eingeschätzt. Natürlich fallen in einem Emissionsszenario, das den Anstieg der globalen Durchschnittstemperatur auf $2^{\circ} \mathrm{C}$ beschränkt, die Risiken geringer aus.

Nordhaus (2010) zeigt in einer Anwendung seines RICEModells, dass eine Reduktion der Emissionen, die den Temperaturanstieg auf $2^{\circ} \mathrm{C}$ beschränkt, zu einem Anstieg der globalen Wohlfahrt (gemessen in Konsumäquivalenzeinheiten) um etwa $19 \%$ führt, im Vergleich zu keiner Emissionskontrolle (und damit einem Temperaturanstieg von $6^{\circ} \mathrm{C}$ ). Allerdings zeigt er auch, dass es optimal wäre, eine weniger restriktive Emissionsvermeidung anzustreben, bei der es zu einem Temperaturanstieg um $3^{\circ} \mathrm{C}$ kommt. Wegen der geringeren Emissionsvermeidungskosten betrüge der Wohlfahrtsgewinn dann $35 \%$. Allerdings basieren diese Schätzungen auf sehr vielen vereinfachenden Annahmen und beinhalten zahlreiche Unsicherheiten über die Entwicklung des Klimawandels, etwa bezüglich des Eintretens von Kipppunkten. Auch potenzielle Einflüsse von Extremereignissen bleiben unberücksichtigt. Kap. 31 diskutiert, zu welchen Ergebnissen diese integrierten Bewertungsmodelle kommen, wenn solche Unsicherheiten explizit berücksichtigt werden. Darüber hinaus ergeben sich selbst bei Analysen, die solche Unsicherheiten nicht berücksichtigen, zahlreiche zu berücksichtigende Szenarien im Hinblick auf die Umsetzung der Emissionskontrolle. Die in Nordhaus (2010) aufgeführten Zahlen basieren auf der Annahme einer international koordinierten Klimaschutzpolitik mit flächendeckendem Handel von Emissionszertifikaten. In der Realität werden die Kosten des Klimaschutzes wahrscheinlich höher ausfallen, wenn nicht alle Staaten die günstigsten Maßnahmen zur Kontrolle der Treibhausgasemissionen einsetzen.

Wirtschaftliche Szenarioanalysen untersuchen detaillierter die sektoralen wirtschaftlichen Auswirkungen und regionalen Risiken. Gleichzeitig sind in den Szenarioanalysen die Kosten (der jetzt exogenen) Emissionskontrolle zu berücksichtigen. Aaheim et al. (2012) untersuchen unter Anwendung des multiregionalen und multisektoralen Wirtschaftsmodells GRACE, mit welchen wirtschaftlichen Auswirkungen die Veränderungen des Klimas in Europa einhergehen werden. Wie in der Darstellung des IPCC berücksichtigen sie Emissionsszenarien, die entweder zu einem Anstieg von 2 oder $4^{\circ} \mathrm{C}$ der globalen Durchschnittstemperatur führen (inklusive der damit verbundenen Vermeidungskosten). Das GRACE-Modell beinhaltet 11 Sektoren, die von den regional unterschiedlichen Veränderungen in Temperatur und Niederschlag beeinflusst werden. Dabei werden auch Aspekte wie die Auswirkungen auf die Arbeitsproduktivität berücksichtigt. Die Autoren schätzen, dass es bei einem Anstieg der globalen Durchschnittstemperatur um $2^{\circ} \mathrm{C}$ nur vergleichsweise moderate Veränderungen im regionalen Bruttoinlandsprodukt (BIP) geben wird und dass einige Regionen sogar etwas profitieren könnten. Bei einem Anstieg der globalen Durchschnittstemperatur um $4^{\circ} \mathrm{C}$ ist zu erwarten, dass alle Regionen in Europa negative wirtschaftliche Auswirkungen verzeichnen könnten, insbesondere der Süden Europas.

Wie schon in der Risikoeinschätzung des Weltklimarats kommt es im Süden von Europa vor allem durch Wasserknappheit zu negativen wirtschaftlichen Auswirkungen, was umgekehrt durch die damit verbundenen Preiseffekte für landwirtschaftli- 
- Tab. 25.1 Überblick über gesamtwirtschaftliche Studien zu den Auswirkungen des Klimawandels in Deutschland

\begin{tabular}{|c|c|c|c|c|c|c|}
\hline Studie & $\begin{array}{l}\text { Methodischer } \\
\text { Ansatz }\end{array}$ & $\begin{array}{l}\text { Klimawan- } \\
\text { delszenario }\end{array}$ & $\begin{array}{l}\text { Betrach- } \\
\text { teter } \\
\text { Zeitraum }\end{array}$ & $\begin{array}{l}\text { Betrachtete Hand- } \\
\text { lungsfelder }\end{array}$ & $\begin{array}{l}\text { Annahmen zum } \\
\text { Stand der Volks- } \\
\text { wirtschaft }\end{array}$ & $\begin{array}{l}\text { Auswirkungen des Klima- } \\
\text { wandels }\end{array}$ \\
\hline $\begin{array}{l}\text { Kemfert } \\
\text { (2007) [nicht } \\
\text { begutachtet] }\end{array}$ & $\begin{array}{l}\text { Top-down-Simu- } \\
\text { lationsmodell } \\
\text { mit sektoralen } \\
\text { Effekten }\end{array}$ & $\begin{array}{l}+4,5^{\circ} \mathrm{C} \text { in } \\
\text { Deutschland } \\
\text { im Jahr } 2100\end{array}$ & $2015-2100$ & $\begin{array}{l}\text { Land- und } \\
\text { Forstwirtschaft, } \\
\text { Tourismus, Ge- } \\
\text { sundheitswesen, } \\
\text { Energie, Verkehr, } \\
\text { Baugewerbe }\end{array}$ & Keine Angaben & $\begin{array}{l}\text { Kumulierte Kosten von } \\
792,5 \text { Mrd. Euro bis } 2050 \text { (da- } \\
\text { von } 296 \text { Mrd. Euro zusätzliche } \\
\text { Energiekosten, 331,5 Mrd. } \\
\text { Euro Schäden, 165,1 Mrd. } \\
\text { Euro Anpassungskosten) }\end{array}$ \\
\hline $\begin{array}{l}\text { Bräuer et al. } \\
\text { (2009) [nicht } \\
\text { begutachtet] }\end{array}$ & $\begin{array}{l}\text { Aufsummierte } \\
\text { sektorale Effekte }\end{array}$ & $\begin{array}{l}+1,5^{\circ} \mathrm{C} \\
{[1,0-1,6]} \\
2{ }^{\circ} \mathrm{C}[1,5-3,5]\end{array}$ & $\begin{array}{l}2050 \\
2100\end{array}$ & $\begin{array}{l}\text { Küsteninfrastruk- } \\
\text { tur, Bauwirtschaft, } \\
\text { Land- und Forst- } \\
\text { wirtschaft, Energie, } \\
\text { Wasserwirtschaft, } \\
\text { Tourismus, Verkehr, } \\
\text { Versicherungen, } \\
\text { Gesundheit }\end{array}$ & $\begin{array}{l}\text { 2011-2050: 1\%, } \\
\text { 2051-2100: 0,5\% } \\
\text { jährliches BIP- } \\
\text { Wachstum }\end{array}$ & $\begin{array}{l}\text { Zwischen }+0,05 \text { und }-0,3 \% \\
\text { des BIP } \\
\text { zwischen }+0,6 \text { und 2,5\% des } \\
\text { BIP als Nettoeffekt auf die } \\
\text { öffentlichen Finanzen }\end{array}$ \\
\hline $\begin{array}{l}\text { Ciscar et al. } \\
\text { (2011) } \\
\text { [begut- } \\
\text { achtet] }\end{array}$ & $\begin{array}{l}\text { Mittels eines ge- } \\
\text { samtwirtschaft- } \\
\text { lichen Modells } \\
\text { sektorale bottom- } \\
\text { up-Modelle } \\
\text { verbunden }\end{array}$ & $\begin{array}{l}2,5^{\circ} \mathrm{C} \\
5,4^{\circ} \mathrm{C}\end{array}$ & 2080 & $\begin{array}{l}\text { Küsteninfrastruktur, } \\
\text { Überschwem- } \\
\text { mungen größerer } \\
\text { europäischer Flüsse, } \\
\text { Landwirtschaft, } \\
\text { Tourismus }\end{array}$ & Stand 2010 & $\begin{array}{l}15 \text { Mrd. Euro BIP } \\
-26 \text { Mrd. Euro BIP für Modell- } \\
\text { region Nördliches Zentraleu- } \\
\text { ropa (Deutschland, Nieder- } \\
\text { lande, Belgien, Polen) }\end{array}$ \\
\hline
\end{tabular}

che Produkte aber nur zu relativ moderaten gesamtwirtschaftlichen Auswirkungen in Zentral- und Osteuropa führt, da hier der landwirtschaftliche Sektor profitiert. Laut der Schätzung von Aaheim et al. (2012) würde es in Deutschland zu Einbußen beim BIP zwischen 0,2 und 0,3\% relativ zu 2004 kommen. Solche vergleichsweise niedrigen aggregierten Auswirkungen sollen aber nicht darüber hinwegtäuschen, dass es sowohl kumuliert als auch insbesondere in einzelnen Sektoren sehr wohl zu deutlich stärkeren Auswirkungen kommen kann. So wird z. B. geschätzt, dass der Forstsektor deutlich stärker beeinträchtigt wird. Außerdem unterschätzt die Studie langfristige Auswirkungen, aber auch den Effekt von langfristigen (geplanten) Anpassungsmaßnahmen.

Die Analyse von Aaheim et al. (2012) ist nur ein Beispiel für Studien, die einen breiteren geografischen Fokus haben und in denen Deutschland nur eine Teilregion darstellt. Im Rahmen des europäischen Forschungsprojekts PESETA wurden bottomup-Schadensmodelle für verschiedene Handlungsfelder entwickelt - Küsteninfrastruktur, Überschwemmungen größerer europäischer Flüsse, Landwirtschaft, Tourismus - und mit einem berechenbaren allgemeinen Gleichgewichtsmodell der europäischen Volkswirtschaft verknüpft. Dabei kommen die Autoren zu dem Schluss, dass sich die Kosten des Klimawandels in Europa in einem Szenario mit einer durchschnittlichen Erwärmung in Europa von $2,5^{\circ} \mathrm{C}$ auf etwa $20 \mathrm{Mrd}$. Euro im Jahr 2080 belaufen werden (Ciscar et al. 2011), wobei gewisse Regionen wie Skandinavien vom Klimawandel profitieren könnten. Würde ein Szenario mit $5,4^{\circ} \mathrm{C}$ Erwärmung und einem unterstellten Anstieg des Meeresspiegels von $88 \mathrm{~cm}$ eintreten, wäre mit jährlichen Kosten in Höhe von 65 Mrd. Euro zu rechnen. Für die Region Nördliches Zentraleuropa, die neben Deutschland auch Belgien, die Niederlande und Polen umfasst, wäre im Szenario mit einer Erwärmung um $2,5^{\circ} \mathrm{C}$ mit Kosten von ungefähr $15 \mathrm{Mrd}$. Euro zu rechnen, die sich im Falle des Szenarios mit starker Erwärmung und hohem
Anstieg des Meeresspiegels auf 26 Mrd. Euro erhöhen würden. Es gibt allerdings nur wenige Studien, die versuchen, die Auswirkungen der komplexen Wirkungsmechanismen im Klimawandel für Deutschland monetär zu bewerten. Drei dieser Studien werden in - Tab. 25.1 zusammengefasst.

Allen drei Studien ist gemeinsam, dass sie einzelne Aspekte des Klimawandels herausgreifen und diese in unterschiedlicher Weise zu gesamtwirtschaftlichen Kosten aggregieren. Während die Analyse von Kemfert $(2007,2008)$ die Aggregation in einem numerischen gesamtwirtschaftlichen Modell vornimmt, werden in Bräuer et al. (2009) nur die Kosten aus den verschiedenen sektoralen Analysen zu einem gesamtwirtschaftlichen Kostenfaktor summiert. Die Ergebnisse des PESETA-Projekts (Ciscar et al. 2011) verknüpfen vergleichsweise detaillierte sektorale Ergebnisse mit einem numerischen allgemeinen Gleichgewichtsmodell, in dem auf einer hohen Aggregationsstufe die Interaktionseffekte simuliert werden.

Kemfert (2007) errechnet mithilfe des globalen Modells WIAGEM kumulierte Kosten des Klimawandels inklusive Kosten der Anpassung von $800 \mathrm{Mrd}$. Euro bis 2050. Das entspricht ungefähr $0,5 \%$ des BIP-Wachstums in der entsprechenden Periode. In einer Nachfolgestudie (Kemfert 2008) wurden diese Ergebnisse auf einzelne Bundesländer heruntergebrochen. Die Simulationen zeigen, dass in absoluten Werten die bevölkerungsreichen und wirtschaftsstarken Bundesländer Baden-Württemberg und Bayern stark betroffen sind. Allerdings werden wirtschaftsschwächere Bundesländer wie Sachsen-Anhalt und Rheinland-Pfalz relativ zu ihrer Bruttowertschöpfung stärker belastet. Die Arbeiten von Kemfert stellen die einzige Analyse dar, die einen klaren Fokus auf Deutschland legt. Allerdings ist anzumerken, dass die hier diskutierten Studien nicht begutachtet wurden und viele Annahmen, gerade bezüglich der Schadensfunktionen, unklar 
und intransparent bleiben. Der Ansatz des Modells WIAGEM wurde beispielsweise von Roson et al. (2006) kritisch diskutiert.

In einer Studie im Auftrag des Bundesministeriums der Finanzen haben Bräuer et al. (2009) die Belastungen infolge des Klimawandels für die öffentlichen Finanzen untersucht. Dabei wurden zehn Handlungsbereiche mittels Fallstudien genauer betrachtet. Die Fallstudien umfassen u.a. Auswirkungen des Klimawandels auf Gebäude, Land- und Forstwirtschaft sowie Energie- und Wasserversorgung. Die Autoren führen keine eigenen Untersuchungen zu Klimafolgen durch, sondern greifen auf bestehende Ergebnisse aus der Literatur zurück und übertragen - sofern nötig - die Ergebnisse auf Deutschland. Dabei werden auf Basis der regionalen Klimamodelle WETTREG und REMO Klimaszenarien für 2050 und 2100 verwendet, die beispielhaft für das Jahr 2050 eine Temperaturänderung von durchschnittlich $1,5^{\circ} \mathrm{C}\left[1,0-1,6^{\circ} \mathrm{C}\right]$, vermehrte Niederschläge im Winter $(+7$ bis $+14 \%)$ und geringere Niederschläge im Sommer (-12 bis $-16 \%)$ beschreiben. Die Schätzungen von Bräuer et al. zeigen, dass 2050 der Klimawandel nur geringe Wirkungen auf die Finanzen der öffentlichen Hand haben könnte. Gemäß der Studie beträgt die zusätzliche Be- oder Entlastung des öffentlichen Haushalts zwischen $+0,1$ und $-0,7 \%$ (relativ zum BIP entspricht das zwischen $+0,05$ und $-0,3 \%$ des BIP). Ab 2100 sind diese Effekte größer - Mehrausgaben und rückläufige Steuereinnahmen könnten zu einer zusätzlichen Belastung zwischen $-1,3$ und $-5,7 \%$ des Haushalts $(-0,6$ und $-2,5 \%$ des BIP) führen. Diese Studie wurde ebenfalls nicht in einer begutachteten Zeitschrift veröffentlicht.

Die Studien von Kemfert und Bräuer bestätigen die weiter oben zitierten Studien mit Fokus auf Europa insofern, als auch sie eher mit geringen wirtschaftlichen Auswirkungen für Deutschland rechnen. Grundsätzlich muss man aber berücksichtigen, dass sich die Arbeiten in fundamentalen Annahmen bezüglich der Struktur der betrachteten Volkswirtschaften, der berücksichtigten Sektoren sowie der Wirkungsketten und -mechanismen der Klimafolgen auf die Ökonomie unterscheiden. So bleibt es z. B. schwierig zu bewerten, inwieweit die globalen Rückkopplungseffekte, die sich durch veränderte Migrations- und Handelsströme ergeben, angemessen berücksichtigt wurden (Schenker 2013). Umgekehrt muss man aber auch berücksichtigen, dass Anpassungsverhalten nicht explizit modelliert wird, sondern meist implizit in den Schadensfunktionen enthalten ist oder als autonome Anpassung durch die Preisreaktionen von Unternehmen und Haushalten berücksichtigt wird. Insofern ist es schwer zu beurteilen, ob diese Studien die Auswirkungen des Klimawandels unter- oder etwa sogar überschätzen.

In einigen empirischen Untersuchungen wird versucht, aus der Analyse des Zusammenhangs zwischen Klimazustand und Wirtschaftswachstum des bestehenden Klimas Regelmäßigkeiten für das Wirtschaftswachstum unter zukünftiger Klimaentwicklung abzuleiten. Diese Studien, welche die Abhängigkeit des Wirtschaftswachstums von Temperatur und Niederschlag in Querschnitts-, Zeitreihen- und Panelschätzungen untersuchen, anstatt die wirtschaftlichen Abläufe explizit zu modellieren, kommen zu dem Schluss, dass Deutschland zu den Profiteuren des Klimawandels gehört. So schätzen z. B. Burke et al. (2015), dass es nur mit einer Wahrscheinlichkeit von $9 \%$ zu einer Verringerung des BIP in Deutschland als Folge des Klimawandels kommen werde und eine deutliche Erhöhung des Pro-Kopf-BIPWachstums wahrscheinlich sei. Natürlich sind solche Studien mit den gleichen bzw. sogar zusätzlichen Einschränkungen zu interpretieren wie die Modelle, die Projektionen für zukünftiges Wirtschaftswachstum vorzunehmen versuchen. Trotzdem liefern empirische Untersuchungen wichtige Hinweise, wie sich z. B. die Arbeitsproduktivität in Abhängigkeit vom Klima entwickeln könnte. Bei der Abschätzung der regionalen gesamtwirtschaftlichen Auswirkungen muss aber kritisch hinterfragt werden, inwieweit die Zusammenhänge zwischen Klima und Wirtschaftswachstum in einem insgesamt wärmeren Klima mit veränderten Waren- und Handelsströmen noch gültig sind. Mit anderen Worten: Die systemischen Änderungen, die in der Weltwirtschaft mit dem Klimawandel einhergehen können, sind hier nicht berücksichtigt. Darüber hinaus bilden aggregierte Veränderungen gemessen in Sozialproduktzahlen nicht die zahlreichen sektoralen und regionalen Herausforderungen und Veränderungen ab, die mit dem Klimawandel einhergehen und zu beträchtlichen Verteilungskonflikten führen können.

\subsection{Abschätzung sektoraler Kosten des Klimawandels}

\subsubsection{Hochwasser- und Küstenschutz}

Die Analyse von Hochwasser- und Küstenschutz hat sich nicht im Rahmen der Anpassungsforschung herausgebildet, sondern ist für sich genommen bereits seit Jahrzehnten Gegenstand wissenschaftlicher Untersuchungen und seit Jahrhunderten gelebte Praxis. Allerdings ergeben sich aus der Dynamik des Klimawandels für die Wissenschaft und die Praxis neue Herausforderungen.

Das Untersuchungsdesign basiert in der Regel auf einer Flutbzw. Überflutungsmodellierung, um die betroffenen Gebiete zu identifizieren. Dann werden mittels verschiedener Schadensfunktionen, beispielsweise in Abhängigkeit von der Landnutzung oder dem vorhandenen Gebäudebestand, die direkten materiellen Schäden ermittelt. Gegebenenfalls werden ergänzend indirekte materielle Schäden abgeleitet, die sich aus dem Verlust von Menschenleben ergeben. Allerdings werden diese Schäden häufig separat betrachtet und nicht in der integrierten Analyse berücksichtigt. Schadensereignisse werden zudem sowohl ohne als auch mit Anpassungsmaßnahmen betrachtet, um den Nutzen von Maßnahmen anhand vermiedener Schäden ableiten zu können. Unsicherheiten in Bezug auf die Ergebnisse resultieren hier vornehmlich aus der Wahl der räumlichen Skala: Je größer diese ist, desto gröbere Annahmen müssen getroffen werden. Je kleiner diese ist, desto detaillierter fallen zwar die Analysen aus, jedoch laufen sie Gefahr, sektorale oder regionale Rückkopplungseffekte und Anpassungsmaßnahmen jenseits des Analyseraums außer Acht zu lassen. Unter den genannten Vorbehalten sind die Ergebnisse einiger jüngerer Studien zu betrachten.

Die PESETA-Studie von Ciscar (2009) unterscheidet fünf Regionen innerhalb der Europäischen Union, wobei Deutschland der Region Nördliches Zentraleuropa zugeordnet ist. Die Bereiche Fluss- und Küstenhochwasser werden separat betrach- 
tet. Bei Flusshochwässern werden für Temperaturanstiege von $2,5^{\circ} \mathrm{C}, 3,9^{\circ} \mathrm{C}, 4,1^{\circ} \mathrm{C}$ und $5,4^{\circ} \mathrm{C}$ im Zeitraum von 2071 bis 2100 deutlich höhere erwartete jährliche Schäden im Vergleich zum simulierten Basiszeitraum von 1961 bis 1990 ermittelt. Sie liegen je nach Szenario zwischen 1,5 Mrd. und 5,3 Mrd. Euro und spiegeln direkte Schäden in Abhängigkeit von der Landnutzung und dem Wasserstand bei Hochwasser wider (Ciscar 2009).

Um die Schäden für verschiedene Meeresspiegelanstiege im Bereich „Küstenhochwasser" zu untersuchen, werden Szenarien mit und ohne Anpassung generiert. Die Landnutzung an den Küsten wird als konstant angenommen. Als Auswirkungen des Klimawandels werden Landverluste und die Zahl der betroffenen Personen betrachtet. In einem bespielhaften Szenario mit starkem Meeresspiegelanstieg $(58,5 \mathrm{~cm})$ ergäbe sich für das nördliche Zentraleuropa ein Verlust von rund 900 Mio. Euro, der den Verlust an produktiver Landfläche widerspiegelt. Bezogen auf das Bruttoinlandsprodukt (BIP) ist der Verlust jedoch sehr klein, denn er liegt bei gut 0,01\%. Er lässt sich zwar durch Anpassung in Form von Küstenschutzinvestitionen noch weiter reduzieren, jedoch aufgrund der indirekten ökonomischen Effekte nicht eliminieren.

Die Arbeit der Policy Research Corporation (2009) analysiert den Status quo des europäischen Küstenschutzes und die aktuellen sowie die geplanten Ausgaben bis 2015. Demnach liegen die Ausgaben der betroffenen Bundesländer in Deutschland pro Jahr bis 2015 bei 49,3 Mio. Euro (Schleswig-Holstein), 30,6 Mio. Euro (Niedersachsen), 27 Mio. Euro (Hamburg), 17,0 Mio. Euro (Mecklenburg-Vorpommern) und 12 Mio. Euro (Bremen). Diese Ausgaben können zugleich als Kosten für die Anpassung interpretiert werden. Betrachtet man die gemäß PESETA-Studie notwendigen Ausgaben, wird konstatiert, dass diese in Deutschland durch die bis 2015 geplanten Ausgaben überschritten werden und demnach aktuell ausreichen müssten.

Rojas et al. (2013) fokussieren auf den Bereich der Flusshochwasser in Europa. Sie differenzieren ihre Ergebnisse dabei nach Ländern. Genutzt wird ein hydrologisches Modell, das zur Schätzung Schadensfunktionen in Abhängigkeit von der Fluthöhe mit Informationen zur Landnutzung und der Bevölkerungsdichte kombiniert. Betrachtet wird ein Klimawandelszenario (SRES A1B), das mit konsistenten Annahmen zum BIP- und Bevölkerungswachstum verbunden wird. Zudem werden Szenarien mit und ohne Anpassung betrachtet. Ermittelt werden nur die direkten Schäden für bestimmte Wiederkehrintervalle. Dabei werden z. B. für ein 100-jährliches Ereignis im Zeitablauf steigende erwartete Schäden berechnet. Sie liegen bei jährlich 540 Mio. Euro (2000er-Jahre), 1,14 Mrd. Euro (2020er-Jahre), 1,38 Mrd. Euro (2050er-Jahre) und 2,92 Mrd. Euro (2080er-Jahre). Auch hier wird Anpassung als lohnende Investition eingeschätzt. Würde in Deutschland eine Anpassung auf ein künftiges 100-jährliches Ereignis erfolgen, so würde dies laut Rojas et al. (2013) Kosten von 170 Mio. Euro verursachen und den erwarteten Schaden deutlich reduzieren.

Aus dem Bereich der grauen Literatur stammt eine Studie, die das PIK gemeinsam mit weiteren Instituten im Auftrag des Gesamtverbandes der Deutschen Versicherungswirtschaft (GDV) erstellt hat, um die mittleren langjährigen Schadensniveaus für Binnenhochwasser zu ermitteln (PIK et al. 2011). Die drei Sze- narien $\mathrm{A} 1 \mathrm{~B}, \mathrm{~A} 2$ und $\mathrm{B} 1$ des Weltklimarats treiben hier regionale Klimamodelle (CCLM, REMO) an, die wiederum Input liefern für das hydrologische Modell SWIM, dass die Hochwasserabflüsse liefert, die schließlich mittels des Modells HQ-Kumul in Überschwemmungsschäden übersetzt werden Als Referenzwert dient der Zeitraum 1961-2000, dem für sieben Modellläufe jeweils die Zeiträume 2011-2040, 2041-2070 und 2071-2100 gegenübergestellt werden. Die Mittelwerte der Modellläufe für die Jahresschäden aller Perioden betragen 0,46, 0,85, 0,89 und 0,99 Mrd. Euro. Es wird also ein recht stetiger Anstieg der zu erwartenden mittleren Schäden festgestellt. Allerdings zeigt sich z. B. in den beiden Regionalmodellen auf Basis des IPCC-Szenarios B1 ein Rückgang der geschätzten Schäden, was die Sensitivität der Resultate verdeutlicht. Zudem nimmt die Bandbreite der Ergebnisse der einzelnen Szenarien im Zeitablauf zu. Weiterhin ist zu bemerken, dass keine sozioökonomischen Änderungen berücksichtigt werden.

Es lässt sich festhalten, dass es für den Bereich des Küstenund Hochwasserschutzes eine Vielzahl an Forschungsprojekten und anderen Anstrengungen gibt, aus denen ein großer Fundus an begutachteter, aber auch grauer Literatur hervorgegangen ist und weiter hervorgeht. Es liegen Analysen auf allen Skalen vor. Dies gilt sowohl hinsichtlich der Schadensschätzung als auch der Bewertung von Anpassungs- und Schutzmaßnahmen. Die zweckmäßige Analyseebene hängt dabei von der Fragestellung ab. So gehen makroskalige Untersuchungen zwar auf Kosten der Detailgenauigkeit, jedoch sind sie eher in der Lage, gesamtwirtschaftliche Effekte und Feedbackmechanismen über Marktprozesse abzubilden. Unzureichend berücksichtigt scheinen in fast allen Studien bisher noch Schäden zu sein, die durch indirekte Effekte hervorgerufen werden, sowie immaterielle Schäden. Indirekte Schäden sind im Status quo zwar im Prinzip modellierbar, hängen in Zukunft aber von sozioökonomischen Veränderungen ab. Immaterielle Schäden unterliegen methodischen Problemen und erfordern in der Bewertung zahlreiche normative Annahmen. Darüber hinaus legen viele der Studien einen starken Fokus auf technische Anpassungsmaßnahmen und Aspekte und die damit verbundenen Kosten. So werden beispielsweise Alternativen wie Umsiedlung und Evakuierung anstelle von verstärktem Küstenschutz kaum berücksichtigt.

\subsubsection{Hitzewellen und Gesundheitskosten}

Der Klimawandel kann eine Reihe von teilweise komplexen Auswirkungen auf die menschliche Gesundheit und Arbeitsproduktivität haben ( Kap. 14). Eine zunehmende Zahl an Studien untersucht die Auswirkungen von Hitzewellen auf die Arbeitsproduktivität. Experimente und Laboruntersuchungen zeigen, dass ab einer Temperatur von $25^{\circ} \mathrm{C}$ die Arbeitsproduktivität mit jedem Grad Temperaturanstieg in etwa um $2 \%$ sinkt (Dell et al. 2014). In einer Metaanalyse zeigen Seppanen et al. (2006), dass ein Temperaturanstieg von 23 auf $30^{\circ} \mathrm{C}$ in etwa mit einem Rückgang der Produktivität um $9 \%$ verbunden ist. Betrachtet man aggregierte Daten auf der Ebene von Wirtschaftssektoren, so zeigt sich wie erwartet, dass unterschiedliche Sektoren unterschiedlich betroffen sind. Graff Zivin und Neidell (2014) 
finden für die USA, dass extrem heiße Tage insbesondere die Arbeitsproduktivität in der Land- und Fortwirtschaft, im Bauund Erdbausektor und in der Energieversorgung beeinträchtigen. Für andere Industriezweige bestätigt sich dieser Einfluss nicht. Es zeigt sich vielmehr, dass Anpassungsmaßnahmen wie verbesserte Klimatisierung die Auswirkungen des Klimawandels für Arbeiten in geschlossenen Räumen begrenzen können. Im Gegensatz zu dieser Studie untersuchen Cachon et al. (2012) ausschließlich den Automobilsektor (wieder in den USA) und zeigen, dass es auch in diesem Sektor mit zahlreichen Arbeiten in klimatisierten Hallen zu Einbußen bei der Arbeitsproduktivität kommt: In Wochen mit extrem heißen Tagen $\left(>32^{\circ} \mathrm{C}\right)$ sinkt die Arbeitsproduktivität um etwa $8 \%$. Inwieweit dieser Effekt durch imperfekte Klimaanlagen, hitzebedingte Effekte außerhalb der Produktionshallen (z. B. verzögerte Anlieferung von Vorprodukten) oder geringere Anwesenheit von Arbeitern erklärt wird, ist unklar. Jones und Olken (2010) betrachten stattdessen Exportdaten und zeigen, dass in armen Ländern (die überwiegend bereits eher hohe Durchschnittstemperaturen haben) ein Anstieg der Temperatur um $1{ }^{\circ} \mathrm{C}$ im Durchschnitt mit einem Rückgang der Exporte um 2,4\% verbunden ist. Dieser Effekt tritt vor allem bei Agrar- und Rohstoffen sowie für Produkte aus dem verarbeitenden Gewerbe auf.

Die Ergebnisse im Hinblick auf die Arbeitsproduktivität lassen sich grundsätzlich auf Deutschland übertragen. Fasst man die Ergebnisse aus dieser Literatur zusammen, ergibt sich eine umgekehrte U-Form für den Zusammenhang zwischen Temperatur und Produktivität bzw. Wachstum (z. B. Nordhaus 2006; Heal und Park 2013; Burke et al. 2015) und damit auch so etwas wie eine „optimale Temperatur" für Produktivität und Wachstum. Da die historische mittlere Temperatur in Deutschland allerdings noch unter dieser optimalen Temperatur liegt, legen diese Studien den Schluss nahe, dass sich im Zuge des Klimawandels die Arbeitsproduktivität in Deutschland erhöht.

Potenzielle Produktivitätseinbußen an sehr heißen Tagen im Sommer würden durch Produktivitätszuwächse im restlichen Jahr überkompensiert. Wie bereits im vorherigen Abschnitt diskutiert, basieren diese Einschätzungen allerdings auf empirischen Zusammenhängen aus dem historischen Klima. Ob steigende Temperaturen in Deutschland zu einer potenziell höheren physischen Arbeitsproduktivität und zu einer Steigerung der gesamtwirtschaftlichen Produktivität führen, hängt auch davon $\mathrm{ab}$, inwieweit Waren- und damit Vorleistungsströme durch den weltweiten Klimawandel beeinflusst werden. Insgesamt bleibt es fraglich, inwieweit solche globalen empirischen Schätzungen zur Prognose geeignet sind.

Ein verändertes Klima mit höheren Temperaturen beeinflusst aber nicht nur direkt die physische Arbeitsproduktivität, sondern auch die Gesundheit und das Wohlbefinden der Menschen (und damit dann auch wieder indirekt die Arbeitsproduktivität). Erhöhte Temperaturen können die Verbreitung neuer Infektionen begünstigen und grundsätzlich die Sterblichkeit erhöhen. Es gibt zwar zahlreiche Untersuchungen im Hinblick auf verringerte oder erhöhte Sterblichkeit durch den Klimawandel, inwieweit sich aber das subjektive Wohlbefinden verändert, ist bislang wenig untersucht ( Kap. 14).
Vor allem weniger belastbare Gruppen wie kranke und alte Menschen sind davon in besonderer Weise betroffen. Neben den internationalen Studien untersuchen Hübler et al. (2008) die Auswirkungen auf Deutschland. Sie bestimmen die volkswirtschaftlichen Kosten von zunehmendem Hitzestress, aber auch reduzierter Kältebelastung anhand der Daten aus dem Hitzejahr 2003. Auf Basis des Emissionsszenarios A1B wurden mittels des regionalen Klimamodells REMO Temperaturverläufe für den Zeitraum 2071-2100 errechnet. Daraus wurde die Veränderung des Ausmaßes hitzebedingter Gesundheitsfolgen abgeleitet, also wie sich Hitze auf die Sterblichkeit, hitzebedingte Krankheiten und die Leistungsfähigkeit auswirkt.

Da die zukünftige weltwirtschaftliche Entwicklung in den letzten Dekaden dieses Jahrhunderts, die Verfügbarkeit von Ressourcen und die Entwicklung des technischen Fortschritts nahezu unbekannt sind, bewerten Hübler et al. (2008) die Gesundheitskosten so, als ob der Klimawandel heute auftreten würde. Sie bestimmen also die Kosten relativ zum heutigen Bruttoinlandsprodukt und zum Stand der wirtschaftlichen Entwicklung. Da die Auswirkungen von Hitzeereignissen anhand der Daten der Hitzewelle 2003 erfolgen, wird unterschätzt, wie stark sich Individuen und Gesellschaft an die größere Gefahr von Hitzewellen nach dem Ereignis im Jahr 2003 angepasst haben und anpassen können. Als Messgröße für die Gesundheitskosten wird die Veränderung hitzebedingter Krankenhauskosten herangezogen, da es über die Kosten ambulanter Behandlungen keine Informationen gab. Daraus resultieren schließlich zusätzliche Krankenhauskosten von 430-500 Mio. Euro pro Jahr für den Prognosezeitraum 2071-2100.

Mithilfe des integrierten Bewertungsmodells WIAGEM und eines Klimaszenarios, das von einer globalen Erwärmung von $4,5^{\circ} \mathrm{C}$ bis zum Jahr 2100 ausgeht, berichtet Kemfert (2007) von zusätzlichen Kosten für den deutschen Gesundheitssektor von $61 \mathrm{Mrd}$. Euro, kumuliert bis 2050. Dabei fallen die Kosten des Klimawandels mit rund $37 \mathrm{Mrd}$. Euro deutlich höher aus als die Anpassungskosten mit 23,8 Mrd. Euro. Allerdings ist unklar, unter welchen Annahmen diese Kosten ermittelt, welche Gesundheitsfolgen dabei genau berücksichtigt und welche Möglichkeiten der Anpassung unterstellt wurden. Während die grundlegenden medizinischen Wirkungsketten zum großen Teil bekannt sind, ist es äußerst schwierig abzuschätzen, wie sich die Gesellschaft an diese Auswirkungen anpassen wird und in welchem ökonomischen und technologischen Umfeld diese Auswirkungen schlussendlich anfallen werden.

Hitze- und/oder Wasserstress beeinflussen aber nicht nur die Arbeitsproduktivität, Gesundheit und Wohlbefinden, sondern haben auch einen unmittelbaren Einfluss auf die politische Stabilität. Hsiang et al. (2013) zeigen im Rahmen einer Metaanalyse, dass Wasserstress und erhöhte Temperaturen zu einem signifikant höheren Konfliktrisiko (zwischen Personen und zwischen Bevölkerungsgruppen) führen. Auch wenn dieser Zusammenhang in Deutschland eher nicht zu erwarten ist, so können doch die Konflikte in anderen Regionen auf Deutschland zurückwirken. Die globale Arbeitsteilung in Form von komplexen Wertschöpfungsketten und durch internationalen Handel können beeinträchtigt werden. Auch direkte Auswirkungen in Form von Migrationsströmen sind zu den mögli- 


\section{Bedarf der Unternehmen an Problemlösungen}

im Jahr 2011, Angaben in Prozent

\begin{tabular}{l} 
Angepasste betriebliche Infrastruktur \\
\hline Versicherung gegen die Folgen von Extremwetterereignissen \\
\hline Sicherstellung der Wasserversorgung \\
\hline Frühwarn- und Informationssysteme \\
\hline Kanalisation und Abwassersysteme \\
\hline Klimaberatung \\
\hline Hochwasserschutz \\
\hline Klimarisikomanagement \\
\hline Lösungen des Kühlwassermangels \\
\hline Sonstige
\end{tabular}

- Abb. 25.3 Unternehmen, für die Klimawandel ein Thema ist, sehen an verschiedenen Stellen Bedarf für Problemlösungen. (Mahammadzadeh et al. 2013) chen, aber kaum monetär zu bewertenden indirekten Kosten des Klimawandels zu rechnen.

\subsection{Exkurs: Subjektive Einschätzung der Betroffenheit von Unternehmen}

Mit einer Reihe von regionalen und bundesweiten Befragungen zu den Wirkungen des Klimawandels auf Unternehmen in Deutschland wurde in den vergangenen Jahren eine empirische Grundlage für die weitere wissenschaftliche Arbeit gelegt (Auerswald und Lehmann 2011; Freimann und Mauritz 2010; Fichter und Stecher 2011; Fichter et al. 2013; IHK 2009; Karczmarzyk und Pfriem 2011; Mahammadzadeh et al. 2013, 2014; Pechan et al. 2011; Stechemesser und Günther 2011). Die Befragungen zeigen, dass dem Klimaschutz derzeit noch weitaus mehr Aufmerksamkeit entgegengebracht wird als der Anpassung an den Klimawandel. Im Hinblick auf die organisatorische Verankerung kann festgestellt werden, dass in den Unternehmen vorwiegend die Einheiten mit dem Klimawandel betraut sind, die sich generell mit Umweltfragen befassen.

Unternehmen erwarten, in wachsendem Maße von den Folgen des Klimawandels betroffen zu sein. Knapp jedes zweite Unternehmen rechnet damit, um das Jahr 2030 durch den Klimawandel negativ betroffen zu sein, sei es im Inland oder im Ausland (Mahammadzadeh et al. 2013). Derzeit sind für Unternehmen die indirekten Folgen von größerem Gewicht als die direkten Folgen, doch werden die direkten Folgen perspektivisch an Bedeutung gewinnen. Auswertungen nach Unternehmensund Funktionsbereichen ergeben, dass die größten Herausforderungen in den Bereichen Logistik sowie Investition und Finanzierung gesehen werden. Mit Blick auf die Risiken aus den direkten Klimafolgen wird insbesondere die betriebliche Logis- tikfunktion als kritisch empfunden. Diese Prozesse sind wettersensibel. Auf solidem Fundament mit Blick auf den Klimawandel stehen die Bereiche Absatz und Vertrieb sowie Personal und Organisation (Mahammadzadeh et al. 2013). Im Branchenvergleich erwarten die Unternehmen der Branchen Maschinenbau und unternehmensnahe Dienstleistungen durch Klimaschutz und Klimaanpassung durchschnittlich mehr Chancen als Risiken und schätzen zudem ihre eigenen Kompetenzen entlang der betrieblichen Wertschöpfungskette eher hoch als gering ein. Auch die Unternehmen der Logistikbranche erhoffen sich durchschnittlich mehr Chancen als Risiken (Mahammadzadeh et al. 2013; Mahammadzadeh 2012).

Rund die Hälfte der Unternehmen sieht für sich aktuell keine direkten Klimafolgen in Deutschland, gut vier Fünftel für sich keine im Ausland (Mahammadzadeh et al. 2013). Nach eigenen Angaben ist für $27 \%$ der Unternehmen, für die der Klimawandel ein Thema ist, der Frost die bedeutsamste Klimafolge. In der Befragung nannten Bauunternehmen den Frost besonders häufig. Dies erklärt sich vermutlich zum Teil durch vergleichsweise kalte Winter vor der Befragung, die im Frühjahr 2011 stattfand. Klimamodelle sagen hingegen für Deutschland mildere Winter voraus. Je nach Region sind dadurch auch häufigere Frost-Tauwetter-Wechsel möglich.

Drei weitere Klimafolgen sind annähernd genauso bedeutsam: Stürme, Starkregenereignisse und Hochwasser sowie der Temperaturanstieg. Eine geringere Bedeutung kommt Hagel, verminderten Niederschlägen im Sommer und Niedrigwasser sowie dem Blitzschlag zu.

Die Befragungen geben auch darüber Auskunft, was einer Anpassung an den Klimawandel seitens der Unternehmen entgegensteht. Als wichtigster Aspekt ist zu nennen, dass sich viele Unternehmen nicht oder noch nicht direkt vom Klimawandel betroffen sehen (Freimann und Mauritz 2010; Mahammadzadeh 
et al. 2013). Eine etwas geringere Rolle spielen indirekte Formen der Betroffenheit. Dabei haben fehlende Marktsignale wie die Nachfrage nach Produkten der Klimaanpassung eine größere Bedeutung als die regulatorische Dimension, bei der es derzeit vor allem Vorschriften zum Klimaschutz und nicht für das weitgehend private Gut der Anpassung an den Klimawandel gibt (Mahammadzadeh et al. 2013). Großes Gewicht kommt nach Angabe der Befragten auch den mit der Klimamodellierung verbundenen Unsicherheiten über den Klimawandel und die Klimafolgen zu.

Unternehmen reagieren bereits heute auf Klimaeinflüsse. Unter den Unternehmen, für die Klimaanpassung und Klimawandel von Bedeutung sind, sind Maßnahmen an Gebäuden mit $60 \%$ am weitesten verbreitet. Das trifft für rund die Hälfte aller Unternehmen zu. In dieser Größenordnung liegen auch die Ergebnisse anderer Unternehmensbefragungen (Fichter und Stecher 2011). Maßnahmen an Gebäuden wie Isolierungen und Verschattungen dienen Klimaanpassung und Klimaschutz gleichermaßen und führen über Energieeinsparungen vergleichsweise schnell zu ökonomischen Gewinnen (Mahammadzadeh et al. 2013 - Abb. 25.3). Von geringerer Bedeutung, aber durchaus noch recht häufig anzutreffen sind der Abschluss von Versicherungen und Maßnahmen im Logistikbereich. Der Befragung von Fichter und Stecher (2011) zufolge beziehen sich die Versicherungslösungen auf Ereignisse wie Stürme und Hagel, aber auch auf Lieferverzögerungen.

Auch wenn die Herausforderungen des sich wandelnden Klimas bei einigen Unternehmen und Branchen bereits Beachtung finden, spielt die Anpassung an den Klimawandel für die Breite der Unternehmen in Deutschland heute noch keine große Rolle. Konkrete Klimaschäden in der Vergangenheit sind ein wesentlicher Anstoß für eigene Initiativen (Osberghaus 2015). Dass die Herausforderungen des Klimawandels auch für das eigene Unternehmen in Zukunft jedoch an Relevanz gewinnen werden, sehen hingegen sehr viele der Befragten (Mahammadzadeh et al. 2013).

\subsection{Kurz gesagt}

Der Klimawandel verändert die Umwelt der Menschen in einer Vielzahl von Aspekten - Temperatur, Niederschläge, Häufigkeit von Extremereignissen, um nur einige Beispiele zu nennen. Er verändert damit auch die biologischen Grundlagen, die Wasserverfügbarkeit, Ökosystemdienstleistungen und nicht zuletzt auch ästhetische Aspekte der Umwelt. Alle diese Veränderungen finden global in unterschiedlichen Ausprägungen statt, verstärken oder verändern sich im Zeitablauf und zeigen ihre Wirkung in sehr unterschiedlichen Zeiträumen, die bis zu mehreren Jahrhunderten und Jahrtausenden reichen.

Die Abschätzung der Kosten dieser systemischen Veränderungen hängt vom zukünftigen Verhalten der Menschheit ab. Das wirtschaftliche Ausmaß des Klimawandels ist zum einen durch die zukünftigen Entscheidungen über die Emission von Treibhausgasen und zum anderen durch die Gestaltung von Anpassungsmaßnahmen bestimmt. Eine vollständige Erfassung und Modellierung dieser Veränderungsprozesse in der Natur und im Handeln von Staaten, Unternehmen und Haushalten über die nächsten Jahrhunderte ist nicht machbar und wird auch in
Zukunft nicht erreicht werden. Heute gibt es einerseits hoch aggregierte Abschätzungen von Klimaschäden, die auf Plausibilitätsüberlegungen beruhen und illustrativen Charakter haben. Andererseits gibt es detaillierte Untersuchungen über die Auswirkungen des Klimawandels auf bestimmte Sektoren oder Handlungsfelder in bestimmten Regionen, die versuchen, die Kosten des Klimawandels realitätsnäher abzuschätzen. Aber auch sie sind mit der Herausforderung konfrontiert, mit großen Unsicherheiten behaftete zukünftige Entwicklungen von Emissionen, Einkommen und Wirtschaftsleistung in ihre Szenarien einbauen zu müssen. Sie können bisher auch nicht die vielfältigen Rückkopplungsprozesse des Klimawandels auf einzelne Volkswirtschaften angemessen abbilden.

Trotzdem haben in den letzten Jahren besonders Studien zu einzelnen Handlungsfeldern die direkten Kosten des Klimawandels in ihren Größenordnungen zu bestimmen versucht. Auch für Europa gibt es einige Projekte und Studien, die die Kosten des Klimawandels abschätzen, aber nur wenige Arbeiten nehmen dabei Deutschland spezifisch in den Blick. Für ausgewählte Sektoren wie den Bereich des Küsten- und Hochwasserschutzes liegt eine Anzahl deutscher Studien vor, aus denen aber nur wenige wissenschaftlich begutachtete Veröffentlichungen entstanden sind. Dabei bleiben meist indirekte Effekte und nichtökonomische, immaterielle Schäden ausgeklammert.

Trotz aller Schwierigkeiten, gesamtwirtschaftliche Kosten des Klimawandels bis zum Ende des Jahrhunderts zu quantifizieren, liefern Szenarienanalysen, empirische Untersuchungen und sektorale Betrachtungen Einschätzungen in Bezug auf die möglichen Kosten. Die verschiedenen Studien lassen den Schluss zu, dass negative Auswirkungen des Klimawandels in Deutschland vor allem durch internationale Rückkopplungseffekte getrieben werden, während ohne diese Effekte die gesamtwirtschaftlichen Auswirkungen sogar positiv sein könnten. Diese Studien bieten aber nur eine grobe Orientierung, weil viele Effekte und Wirkungskanäle noch nicht ausreichend untersucht sind. Nichtsdestotrotz zeigen die Studien durch den Vergleich verschiedener Emissionsszenarien die Bedeutung des Klimaschutzes sowie insgesamt die Bedeutung der Anpassung für die Begrenzung der Kosten des Klimawandels auf.

\section{Literatur}

Aaheim A, Amundsen H, Dokken T, Wei T (2012) Impacts and adaptation to climate change in European economies. Glob Environ Chang 22(4):959-968

Auerswald H, Lehmann R (2011) Auswirkungen des Klimawandels auf das Verarbeitende Gewerbe - Ergebnisse einer Unternehmensbefragung. Ifo Dresden berichtet 2/2011:16-22

Bosello F, Carraro C, De Cian E (2010) Climate policy and the optimal balance between mitigation, adaptation, and unavoided damage. Clim Chang Econ 1:71-92

Bräuer I, Umpfenbach K, Blobel D et al (2009) Klimawandel: Welche Belastungen entstehen für die Tragfähigkeit der Öffentlichen Finanzen? Ecologic Institute, Berlin. http://ecologic.eu/download/projekte/1850-1899/1865/ Endbericht_FINAL_Klimawandel.pdf

Bruin K de, Dellink R, Tol R (2009) AD-DICE: an implementation of adaptation in the DICE model. Clim Chang 95:63-81

Burke M, Hsiang SM, Miguel E (2015) Global non-linear effect of temperature on economic production. Nature 527:235-239 
Cachon G, Gallino S, Olivares M (2012) Severe Weather and Automobile Assembly Productivity. The Wharton School, University of Pennsylvania. http:// opim.wharton.upenn.edu/ cachon/pdf/weather_1015.pdf

Ciscar JC (2009) Climate change impacts in Europe. Final report of the PESETA research project. Joint Research Centre Scientific and Technical Report. http://ipts.jrc.ec.europa.eu/publications/pub.cfm?id=2879

Ciscar JC, Iglesias A, Feyen L et al (2011) Physical and economic consequences of climate change in Europe. Proceedings of the National Academy of Sciences 108:2678-2683

Dell M, Jones BF, Olken BA (2014) What do we learn from the weather? The new climate-economy literature. J Econ Lit 52:740-798

Fichter K, Stecher T (2011) Wie Unternehmen den Folgen des Klimawandels begegnen. Chancen und Risiken der Anpassung an den Klimawandel aus Sicht von Unternehmen der Metropolregion Bremen-Oldenburg, 13. Werkstattbericht. Universität Oldenburg, Oldenburg

Fichter K, Hintemann R, Schneider T (2013) Unternehmensstrategien im Klimawandel. Fallstudien zum strategischen Umgang von Unternehmen mit den Herausforderungen der Anpassung an den Klimawandel, 20. Werkstattbericht. Universität Oldenburg, Oldenburg

Fisher-Vanden K, Wing SI, Lanzi E, Popp D (2013) Modeling climate change feedbacks and adaptation responses: recent approaches and shortcomings. Clim Chang 117:481-495

Freimann J, Mauritz C (2010) Klimawandel und Klimaanpassung in der Wahrnehmung unternehmerischer Akteure. Werkstattreihe Nachhaltige Unternehmensführung, Bd. 26. Universität Kassel, Kassel

Graff Zivin J, Neidell MJ (2014) Temperature and the allocation of time: implications for climate change. J Labor Econ 32(1):1-26

Heal und Park 2013 Feeling the Heat:Temperature, Physiology \& the Wealth of Nations NBER Working Paper No. 19725

Hsiang M, Solomon M, Burke M, Miguel E (2013) Quantifying the influence of climate on human conflict. Science 341:6151

Hübler M, Klepper G, Peterson S (2008) Costs of climate change - the effects of rising temperatures on health and productivity in Germany. Ecol Econ 68(1-2):381-393

IHK - Industrie- und Handelskammer für München und Oberbayern (2009) Die Wirtschaft und der Klimawandel. Reaktionen der Unternehmen, Studie in Zusammenarbeit mit dem Bayerischen Staatsministerium für Umwelt und Gesundheit. Industrie- und Handelskammer für München und Oberbayern, München

IPCC (2014) Summary for policymakers. Climate change 2014: Impacts, adaptation, and vulnerability. Part A: Global and sectoral aspects. In: Field CB, Barros VR, Dokken DJ, Mach KJ, Mastrandrea MD, Bilir TE, Chatterjee M, Ebi KL, Estrada YO, Genova RC, Girma B, Kissel ES, Levy AN, MacCracken S, Mastrandrea PR, White LL (Hrsg) Contribution of Working Group II to the Fifth Assessment Report of the Intergovernmental Panel on Climate Change. Cambridge University Press, Cambridge, S 1-32

Jones BF, Olken BA (2010) Climate shocks and exports. Am Econ Review 100(2):454-459

Karczmarzyk A, Pfriem R (2011) Klimaanpassungsstrategien von Unternehmen. Metropolis Verlag, Marburg

Kemfert C (2007) Klimawandel kostet die deutsche Volkswirtschaft Milliarden. DIW Wochenbericht 74(11):165-170

Kemfert C (2008) Kosten des Klimawandels ungleich verteilt: Wirtschaftsschwache Bundesländer trifft es am härtesten. DIW Wochenbericht 75(11):147142

Mahammadzadeh M (2012) Klimaschutz und Klimaanpassung in Unternehmen: Eine SWOT-analytische Betrachtung der betrieblichen Funktionen. uwf UmweltWirtschaftsForum 20(2-4):165-173

Mahammadzadeh M, Chrischilles E, Biebeler H (2013) Klimaanpassung in Unternehmen und Kommunen. Betroffenheiten, Verletzlichkeiten und Anpassungsbedarf. IW-Analysen, Bd. 83. Forschungsberichte aus dem Institut der deutschen Wirtschaft, Köln

Mahammadzadeh M, Bardt H, Biebeler H, Chrischilles E, Striebeck J (2014) Anpassung an den Klimawandel von Unternehmen - Theoretische Zugänge und empirische Befunde. Oekom Verlag München

Nordhaus W (2014) Estimates of the social cost of carbon: concepts and results from the DICE-2013R model and alternative approaches. J Assoc Environ Res Econ 1(1/2):273-312
Nordhaus WD (1991) To slow or not to slow: the economics of the greenhouse effect. The Econ J 101:920-937

Nordhaus WD (2006) Geography and macroeconomics: new data and new findings. PNAS 103(10):3510-3517

Nordhaus WD (2010) Economic aspects of global warming in a post-Copenhagen environment. Proceedings of the National Academy of Sciences 107:11721-11726

Nordhaus WD, Yang Z (1996) A regional dynamic general-equilibrium model of alternative climate-change strategies. Am Econ Rev 86:741-765

O'Neill BC et al (2013) A new scenario framework for climate change research: the concept of shared socio-economic pathways. Clim Chang 122(3):1-14

Osberghaus D (2015) The determinants of private flood mitigation measures in Germany - evidence from a nationwide survey. Ecol Econ 110:36-50

Pechan A, Rotter M, Eisenack K (2011) Anpassung in der Versorgungswirtschaft. Empirische Befunde und Einflussfaktoren. In: Karczmarzyk A, Pfriem R (Hrsg) Klimaanpassungsstrategien von Unternehmen. Metropolis Verlag, Marburg, S 313-335

PIK, FUB, UK, IAWG (2011) Auswirkungen des Klimawandels auf die Schadensituation in der deutschen Versicherungswirtschaft; Studie im Auftrag des GDV. http://www.gdv.de/wp-content/uploads/2012/01/Klimakonferenz_2011_PIK_Studie_Hochwasser.pdf

Pindyck RS (2013) Climate Change Policy: What Do the Models Tell Us? J Econ Lit 51:860-872

Policy Research Corporation (PRC) (2009) The economics of climate change adaptation in EU coastal areas, on behalf of the European Commission. http://ec.europa.eu/maritimeaffairs/documentation/studies/climate_ change_en.htm

Rojas R, Feyen L, Watkiss P (2013) Climate change and river floods in the European Union: socio-economic consequences and the costs and benefits of adaptation. Glob Environ Chang 23(6):1737-1751. doi:10.1016/j.gloenvcha.2013.08.006

Roson R et al (2006) An integrated assessment model of economy-energyclimate-the model Wiagem: a comment. Integr Assess J 6(1):75-82

Schenker O (2013) Exchanging goods and damages: the role of trade on the distribution of climate change costs. Environ Res Econ 54(2):261-282

Seppanen O, Fisk WJ, Lei Q (2006) Effect of temperature on task performance in office environment. Ernest Orlando Lawrence Berkeley National Laboratory, Berkeley

Stechemesser K, Günther E (2011) Herausforderung Klimawandel, Auswertung einer deutschlandweiten Befragung im Verarbeitenden Gewerbe. In: Karczmarzyk A, Pfriem R (Hrsg) Klimaanpassungsstrategien von Unternehmen. Metropolis Verlag, Marburg, S 59-83

Stern (2007) The economics of climate change: the Stern review. Cambridge University Press, Cambridge

Vuuren DP van, Stehfest E, Elzen MGJ, Kram T, Vliet J, Deetman S, Isaac M, Goldewijk K, Hof A, Beltran MA, Oostenrijk R, Ruijven B (2011) RCP2.6 Exploring the possibility to keep global mean temperature increase below $2^{\circ} \mathrm{C}$. Clim Chang 109(1-2):95-116

Open Access Dieses Kapitel wird unter der Creative Commons Namensnennung 4.0 International Lizenz (http://creativecommons.org/ licenses/by/4.0/deed.de) veröffentlicht, welche die Nutzung, Vervielfältigung, Bearbeitung, Verbreitung und Wiedergabe in jeglichem Medium und Format erlaubt, sofern Sie den/die ursprünglichen Autor(en) und die Quelle ordnungsgemäß nennen, einen Link zur Creative Commons Lizenz beifügen und angeben, ob Änderungen vorgenommen wurden.

Etwaige Abbildungen oder sonstiges Drittmaterial unterliegen ebenfalls der genannten Creative Commons Lizenz, sofern sich aus der Abbildungslegende oder der Quellreferenz nichts anderes ergibt. Sofern solches Drittmaterial nicht unter der genannten Creative Commons Lizenz steht, ist eine Vervielfältigung, Bearbeitung oder öffentliche Wiedergabe nur mit vorheriger Zustimmung des betreffenden Rechteinhabers oder auf der Grundlage einschlägiger gesetzlicher Erlaubnisvorschriften zulässig. 